\title{
AMMECR1 Inhibits Apoptosis and Promotes Cell-cycle Progression and Proliferation of the A549 Human Lung Cancer Cell Line
}

\author{
HAIBO GE ${ }^{1 *}$, NINGCHANG CHENG ${ }^{2 *}$, XIAOFENG XU ${ }^{3 *}$, ZHIJIAN YANG ${ }^{4,5}$, \\ ROBERT M. HOFFMAN ${ }^{5,6}$ and JIA ZHU ${ }^{7}$ \\ ${ }^{1}$ Department of Respiratory Medicine, Third Affiliated Hospital of Nanjing \\ University of Chinese Medicine, Nanjing, P.R. China; \\ ${ }^{2}$ Xinglong Community Health Service Center, Nanjing, P.R. China; \\ ${ }^{3}$ Department of Respiratory Medicine, Nanjing Chest Hospital, Nanjing, P.R. China; \\ ${ }^{4}$ Origin Biosciences, Nanjing, P.R. China; \\ ${ }^{5}$ AntiCancer Inc. San Diego, CA, U.S.A.; \\ ${ }^{6}$ Department of Surgery, University of California, San Diego, CA, U.S.A.; \\ ${ }^{7}$ Department of Respiratory Medicine, Affiliated Hospital of Nanjing \\ University of Chinese Medicine, Nanjing, P.R. China
}

\begin{abstract}
Aim: The aim of this study was to characterize the role of Alport syndrome, mental retardation, midface hypoplasia, and elliptocytosis chromosomal region gene 1 (AMMECR1) in human lung cancer cell lines. Materials and Methods: AMMECR1 gene expression was evaluated in four lung cell lines, with A549 then selected for further in-depth examination. To characterize the role of AMMECR1, silencing was achieved utilizing lentivirus-mediated RNA interference, and confirmed by quantitative real-time polymerase chain reaction and western blotting. The impact of AMMECRI silencing on cellular proliferation was assessed using Celigobased and MTT assays. Apoptosis was determined using the annexin V-allophycocyanin single staining method. Cell-cycle arrest was assessed by flow cytometry. Finally, colony formation was assessed using Giemsa staining. Results: In A549 cells, AMMECRI silencing was found to significantly suppress cell proliferation, reduce colony formation, promote apoptosis, and arrest cells in the $S$ and $G_{2} / M$ phases. Conclusion: AMMECRI plays a critical role in cell proliferation, cell-cycle progression,
\end{abstract}

This article is freely accessible online.

*These Authors contributed equally to this study.

Correspondence to: Jia Zhu, Department of Respiratory Medicine, Affiliated Hospital of Nanjing University of Chinese Medicine, 155 Hanzhong Road, Nanjing, Jiangsu Sheng 210006, P.R. China. E-mail: jsnjzj@163.com

Key Words: Lung cancer cell, AMMMECR1, cell cycle, cell proliferation, apoptosis. and apoptosis of human lung cancer cells, and may serve as a potential therapeutic target for non-small-cell lung cancer.

Lung cancer is one of the most prevalent and deadly malignancies in the world (1). Improvements in diagnosis and therapy have substantially extended the survival of patients with lung cancer (2). However, most patients experience recurrences within 5 years, with limited therapeutic options $(3,4)$. Precise molecular characterization of abnormal gene expression involved in lung cancer development and progression is necessary to identify novel molecular targets of non-small-cell lung cancer (NSCLC) which may improve clinical outcome in the future. In Homo sapiens, Alport syndrome, mental retardation, midface hypoplasia, and elliptocytosis chromosomal region gene 1 $(A M M E C R l)$ is highly conserved, but its function is poorly understood (5). Deletion of AMMECRI induced the AMME complex, which is a contiguous gene deletion syndrome $(6,7)$. Furthermore, $A M M E C R 1$ has been shown to be frequently expressed in the K-562 (myeloid) and NTERA-2 cell lines (The Human Protein Atlas, http://www.proteinaltas.org/search/ AMMECR1). Kaplan-Meier analysis has demonstrated that lower $A M M E C R 1$ expression is associated with higher overall survival in patients with gastric cancer $(p<0.01)$, but with lower overall survival in patients with ovarian and lung cancer (http://kmplot.com/ analysis/). However, the role of AMMECRI in lung cancer is still not fully understood.

In the present study we examined $A M M E C R l$ expression in several lung cancer cell lines. AMMECRl knockdown in lung cancer cells was performed to investigate the role of $A M M E C R l$ in lung cancer cell proliferation, apoptosis and the cell-cycle. 


\section{Materials and Methods}

Cell culture. Human lung cancer cell line A549, NCI-H1299, NCIH1975 and NCI-H460 as well as human bronchial epithelial cell line HBE were obtained from the Shanghai Cell Bank of the Chinese Academy of Science (Shanghai, PR China). The cells were cultured in Dulbecco's modified Eagle's medium containing $10 \%$ fetal calf serum, $100 \mu \mathrm{g} / \mathrm{ml}$ streptomycin, and $100 \mathrm{U} / \mathrm{ml}$ penicillin, and incubated at $37^{\circ} \mathrm{C}$ with $5 \% \mathrm{CO}_{2}$.

Cell transfection. Short hairpin RNA (shRNA) targeting AMMECRI (H. sapiens; Accession\#: NM_015365) was designed and the following sequence was generated: 5'-CCG GGA GGA TAC AAA GCT CCG ATT ACT CGA GTA ATC GGA GCT TTG TAT CCT CTT TTT G-3'. A negative control shRNA sequence was also generated as follows: 5'-AAT TCA AAA AGA GGA TAC AAA GCT CCG ATT ACT CGA GTA ATC GGA GCT TTG TAT CCT C-3'. Double-stranded DNA oligonucleotides were synthesized based on the shRNA sequences and inserted into lentiviral vectors (GV115; Jikai Gene Chemical Technology Co., Ltd., Shanghai, PR China) with AgeI/EcoRI sites. A549 cells were transfected with the shAMMECR 1 lentivirus or with negative control lentivirus (shCtrl) according to the manufacturer's instructions. The transfected cells were then observed under fluorescence microscopy (Olympus, Tokyo, Japan) at $72 \mathrm{~h}$ transfection. Transfection efficiency was confirmed based on downregulation of AMMECR1 expression determined by real-time polymerase chain reaction (PCR) and blotting analysis.

Quantitative real-time polymerase chain reaction ( $q P C R)$. Parental A549, NCI-H1299, NCI-H1975, NCI-H460 and HBE cells were harvested when the cultured cells reached $80 \%$ confluence. The transfected A549 cells were harvested when the cells were cultured for $72 \mathrm{~h}$ to $80 \%$ confluence. Total RNA was extracted from parental or transfected A549 cells using TRIzol reagent (Shanghai Pufei Biotechnology, Co., Ltd., Shanghai, PR China). RNA concentration and purity were assessed using a NanoDrop ND-1000 spectrophotometer (Thermo Fisher Scientific, Waltham, MA, USA). Approximately $2 \mu \mathrm{g}$ total RNA was reverse-transcribed using a M-MLV reverse transcriptase kit (Promega, Madison, WI, USA) according to the manufacturer's instructions, followed by PCR amplification with specific AMMECR1 primers: 5'-AACTGACCAGGTATCGTAGTG-3' (forward) and 5'-GGGATGCCC AATGCCATTTTG-3' (reverse). Glyceraldehyde 3-phosphate dehydrogenase $(G A P D H)$ was used as an endogenous control with the following primers: 5'-TGACTTCAACAG CGACACCCA-3' (forward) and 5'-CACCCTGTTGCTGTAGCCA AA-3' (reverse). The AMMECR1 and GAPDH PCR products were 98 and $121 \mathrm{bp}$, respectively. The $2^{-\Delta \Delta \mathrm{Ct}}$ method was used to determine the relative mRNA expression level. All reactions were repeated three times for each sample.

Western blot analysis. The transfected A549 cells were harvested after culture for $72 \mathrm{~h}$ to $80 \%$ confluence. The cells then were washed with phosphate-buffered saline, and lysed using ice-cold $2 \times$ lysis buffer (100 mM Tris-HCl, 0.2\% $\beta$-mercaptoethanol, $0.1 \%$ glycerol, and $0.1 \%$ sodium dodecylsulfate). Total proteins were separated by sodium dodecylsulfate-polyacrylamide gel electrophoresis and electrophoretically transferred to a polyvinylidene fluoride membrane (Millipore, Bedford, MA, USA). The membranes were incubated with primary antibody to AMMECR1 or GAPDH (Santa Cruz Biotechnology, Santa Cruz, CA, USA).
After washing twice with Tris-buffered saline, the membranes were incubated with appropriate secondary antibody conjugated with horseradish peroxidase (Ausbian, Shanghai, PR China). Electrochemiluminescence was performed according to the manufacturer's instructions using enhanced chemiluminescence (Thermo, Shanghai, PR China).

Cell proliferation assay. shAMMECR1-transfected and shCtrltransfected cells were seeded in 96-well plates at a density of 2,000 cells $/ \mu \mathrm{l}$ per well and cultured at $37^{\circ} \mathrm{C}$ in $5 \% \mathrm{CO}_{2}$. Green fluorescent protein (GFP) was the marker of lentivirus-transfected cells (8). GFP-expressing cells in each well were counted at different time points with a Cellomics ArrayScan VT1 Reader (Cellomics, Inc., Pittsburgh, PA, USA). The cell proliferation curve was plotted.

3-(4,5-Dimethylthiazol-2-yl)-2,5-diphenyltetrazolium bromide (MTT) assay. shAMMECR1 and shCtrl cells were seeded in 96-well plates with $5 \times 10^{3 / 200} \mu \mathrm{I}$ and cultured for 5 days. To measure cell viability, $10 \mu 1$ 3-(4,5-dimethylthiazol-2-yl)-2,5-diphenyltetrazolium bromide (MTT) $(5 \mu \mathrm{g} / \mu \mathrm{l})$ (ATCC, Manassas, VA USA) was added to each well and incubated for $4 \mathrm{~h}$ at $37^{\circ} \mathrm{C}$. dimethyl sulfoxide (ShiYi Pharmaceutical Group, Shanghai, PR China) $(100 \mu \mathrm{l})$ was then added. After 10 min shaking, cell proliferation was measured at $\mathrm{OD}_{490}$ using a microplate reader (Tecan, Durham, NC, USA).

Apoptosis assay. To determine apoptosis levels, the annexin V-allophycocyanin (APC) single-cell staining method was utilized (9). shAMMECR1 and shCtrl cells were collected and washed twice with $4^{\circ} \mathrm{C}$ pre-cooled D-Hanks solution (Jikai Gene Chemical Technology Co., Ltd., Shanghai, PR China) (pH 7.2-7.4). Annexin V-APC reagent (Abcam, Shanghai, China) $(10 \mu \mathrm{l})$ was added and cells were incubated in the dark at room temperature for $10 \mathrm{~min}$. Flow cytometric analysis was performed on a fluorescenceactivated cell sorting (FACS) Calibur platform (Guava easyCyte HT; Millipore).

Cell-cycle assay. Cell-cycle phase distribution was measured by staining DNA with propidium iodide. shAMMECR1-transfected and shCtrl-transfected cells were seeded in 6-well plates and cultured at $37^{\circ} \mathrm{C}$ and $5 \% \mathrm{CO}_{2}$. When the cells reached $80 \%$ confluency, they were harvested and fixed in $70 \%$ ethanol (precooled $4^{\circ} \mathrm{C}$ ) for at least $1 \mathrm{~h}$. Then the cells were centrifuged at $300 \times g$ for $5 \mathrm{~min}$, and washed with precooled D-Hanks $\left(4^{\circ} \mathrm{C} ; \mathrm{pH}\right.$ 7.2-7.4). Based on the cell count, 0.6-1 ml cell-staining solution (40× propidium iodide, $2 \mathrm{mg} / \mathrm{ml}$; Sigma-Aldrich, Saint Louis, MO, USA), $100 \times$ RNase A solution (10 mg/ml; Fermentas, Belgium) and $1 \times$ D-Hanks were added at a ratio of 25:10:1000. The cell suspension was analyzed using a FACS Calibur platform (Guava easyCyte HT) at a flow rate of 300-800 cells/s.

Colony-formation assay. shAMMECR1-transfected and shCtrltransfected cells were seeded in 6-well plates at a density of 4001,000 cells per well. The cells were then cultured and observed over 14 d. Cell colonies were imaged by GFP expression with a fluorescence microscope (Olympus IX71; Olympus, Japan). At the end of the culture period, the cells were washed with phosphatebuffered saline, and stained with Giemsa (Shanghai Dingguo Biotech Co., Ltd., Shanghai, PR China). After washing with doubledistilled water, the cells were allowed to dry and were then imaged to obtain colony counts. 


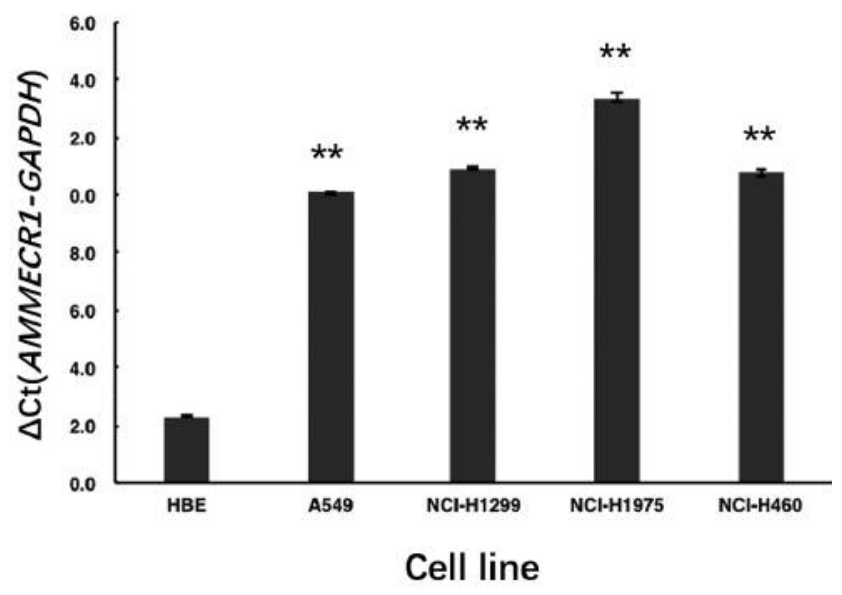

Figure 1. Expression of Alport syndrome, mental retardation, midface hypoplasia, and elliptocytosis chromosomal region gene 1 (AMMECR1) was elevated in lung cancer cell lines compared to a normal cell line. AMMECRI expression level was examined in lung cancer cell lines A549, NCI-H1299, NCI-H1975, NCI-H460 and human bronchial epithelial cell line HBE using quantitative polymerase chain reaction. AMMECR1 was significantly elevated in all four human lung cancer cell lines compared to HBE cells. ${ }^{*}$ Significantly different at $p<0.01$.

Statistical analysis. All data are presented as mean values \pm standard deviation and were analyzed using SPSS 19 (SPSS, Chicago, IL, USA). Comparisons between groups were performed using Student's $t$-test. $p$-Values of less than 0.05 are considered to indicate a statistically-significant difference.

\section{Results}

AMMECRI expression is elevated in human lung cancer cell lines. AMMECR1 mRNA expression was assessed in the A549, NCI-H1299, NCI-H1975 and NCI-H460 lung cancer cell lines and human bronchial epithelial cell line HBE via qPCR. mRNA expression of AMMECRI was significantly elevated in all four human lung cancer cell lines compared to HBE cells $(p<0.01)$ (Figure 1). Since the difference in AMMECR1 mRNA expression was not significant amongst the four cancer cell lines, we selected the commonly-used A549 cell line for subsequent studies.

Knockdown of AMMECR1 reduces AMMECR1 expression in A549 cells. AMMECR1 knockdown was performed by transfecting A549 cells with AMMECR-specific shRNA. $A M M E C R I$ mRNA expression was significantly inhibited in shAMMECR 1-transfected cells compared to shCtrltransfected cells (Figure 2A). Furthermore, western blot analysis showed that AMMECR 1 protein expression was reduced in shAMMECR1-transfected cells compared to shCtrl-transfected cells (Figure 2B).

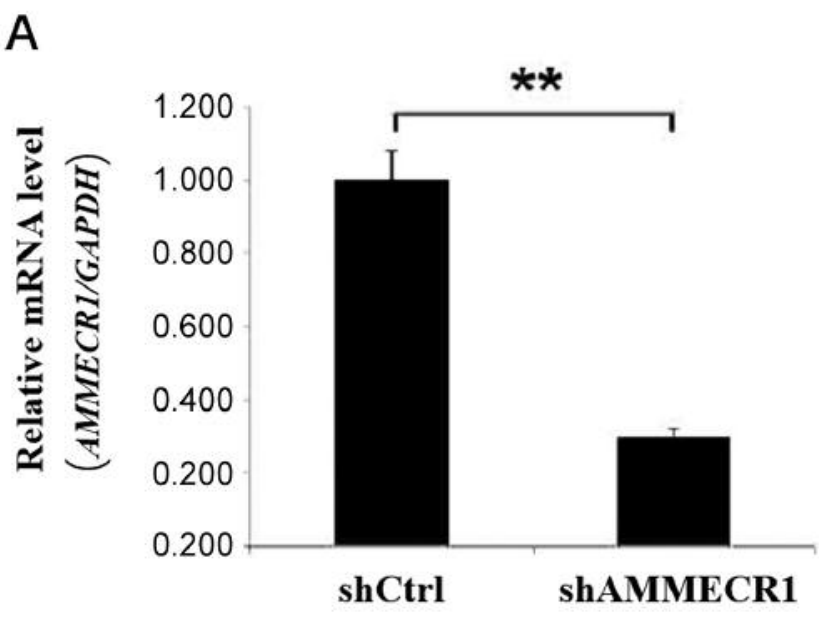

B

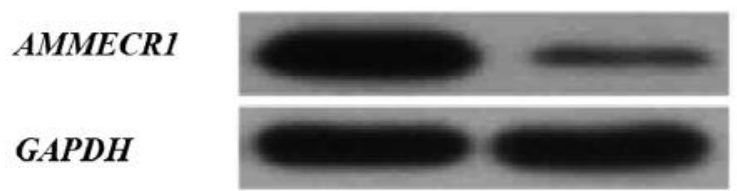

Figure 2. Knockdown of Alport syndrome, mental retardation, midface hypoplasia, and elliptocytosis chromosomal region gene 1 (AMMECR1) with specific short hairpin RNA (shAMMECR1) which reduced AMMECR1 expression in A549 cells. A: AMMECR1 mRNA expression was significantly inhibited in shAMMECR1-transfected cells compared to shCtrl-transfected cells. B: AMMECR1 protein expression was significantly inhibited by transfection with shAMMECR1 compared to shCtrl-transfected cells. GAPDH: Glyceraldehyde 3-phosphate dehydrogenase. **Significantly different at $p<0.01$.

AMMECRI knockdown inhibits cell proliferation in A549 cells. Effect of AMMECRl knockdown on cell proliferation was examined using Cellomics ArrayScan VTI imaging. As shown in Figure 3, cell proliferation was significantly inhibited in shAMMECR1-transfected cells compared to shCtrl-transfected cells $(p<0.01)$. This finding was further confirmed using the MTT assay, which also demonstrated a significant reduction in cell proliferation in shAMMECR1-transfected cells compared to shCtrl-transfected cells $(p<0.01)$ (Figure 4).

AMMECR1 knockdown increases apoptosis in A549 cells. Cell apoptosis was determined using an annexin V-APC assay. A significant increase in apoptosis was found in shAMMECR1-transfected cells compared to shCtrltransfected cells $(p<0.01)$ (Figure 5).

AMMECR1 knockdown leads to cell-cycle arrest in A549 cells. As shown in Figure 6, transfection with shAMMECR1 led to a significant decrease in the 


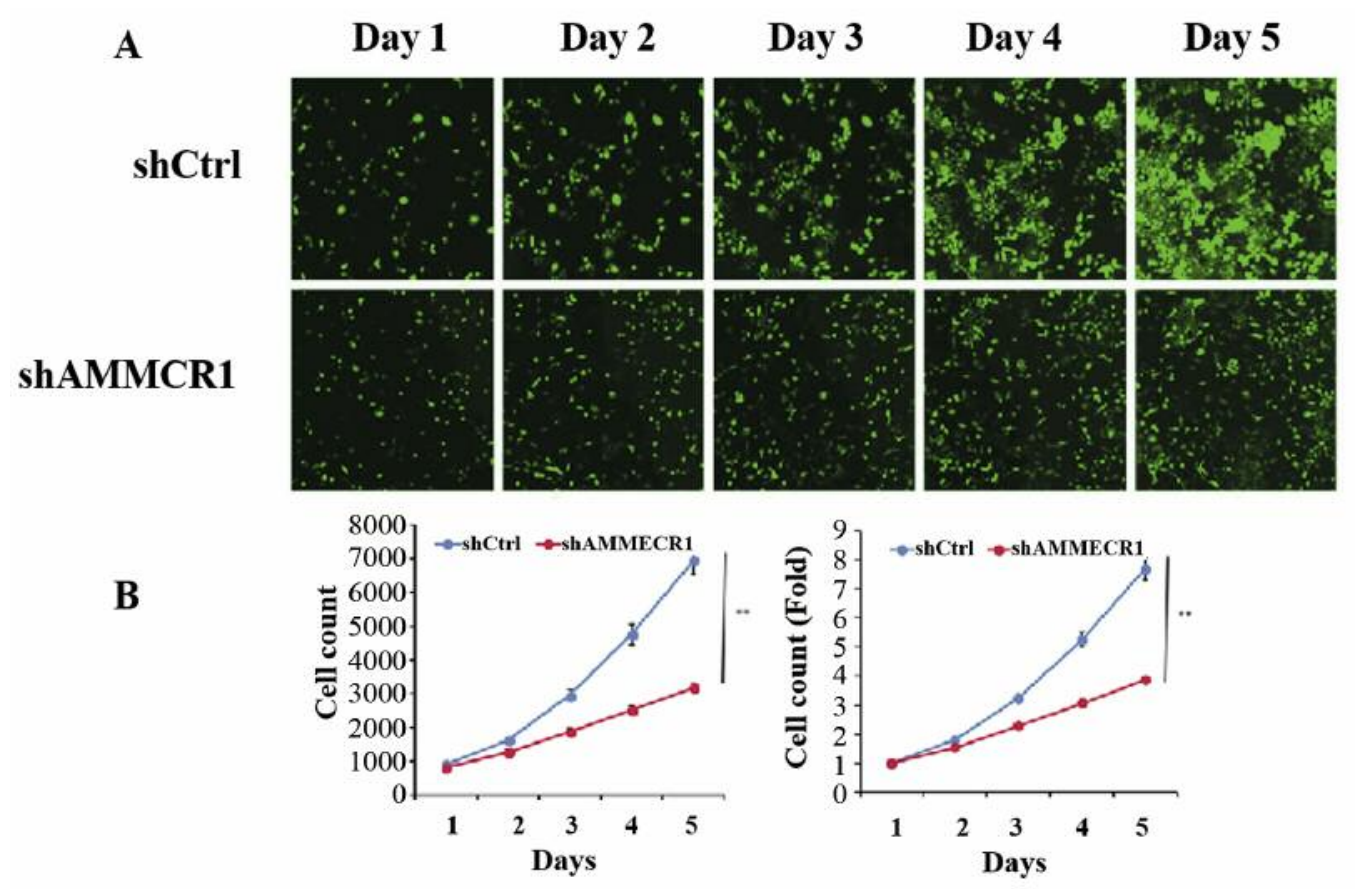

Figure 3. Knockdown of Alport syndrome, mental retardation, midface hypoplasia, and elliptocytosis chromosomal region gene 1 (AMMECR1) inhibited proliferation of A549 cells. A: Fluorescence imaging of cells transfected with AMMECR1-specific short hairpin RNA (shAMMECR1) and shCtrl at different time points (magnification, $\times 100$ ). B: Cell count with a Cellomics ArrayScan VTI Reader over a period of 5 days. **Significantly different from shCtrl-transfected cells at $p<0.01$.
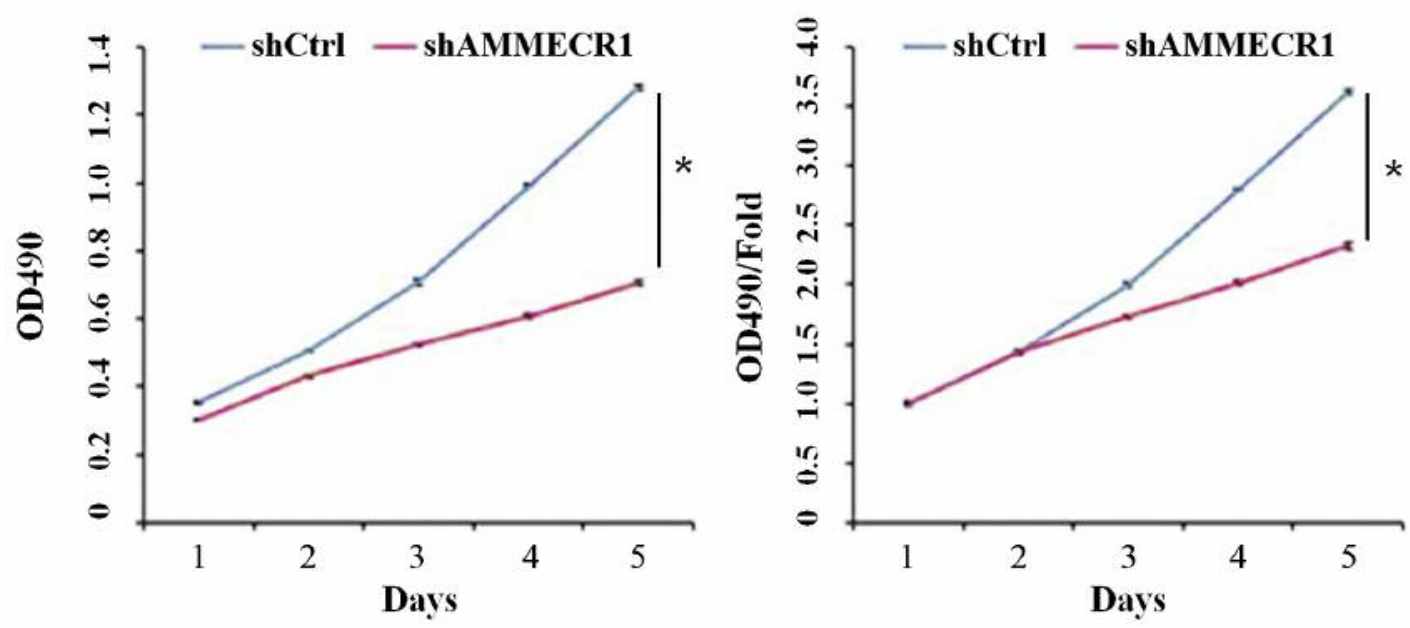

Figure 4. 3-(4,5-Dimethylthiazol-2-yl)-2,5-diphenyltetrazolium bromide (MTT) assay for cell proliferation. Alport syndrome, mental retardation, midface hypoplasia, and elliptocytosis chromosomal region gene 1-specific short hairpin RNA (shAMMECR1)-transfected cells showed significantly lower proliferation compared to shCtrl transfected cells. *Significantly different from shCtrl-transfected cells at $p<0.05$.

percentage of cells in the $S$ phase and $\mathrm{G}_{2} / \mathrm{M}$ phase compared to shCtrl-transfected cells $(p<0.01)$. The percentage of cells in $\mathrm{G}_{1}$ phase was significantly increased in shAMMECRl-transfected cells compared to shCtrltransfected cells $(p<0.01)$.
AMMECR1 knockdown reduced colony formation by A549 cells. As shown in Figure 7, transfection with shAMMECR 1 significantly reduced cell-colony formation compared to shCtrl-transfected cells $(p<0.01)$. These results suggest that AMMECRI promotes proliferation of A549 cells. 


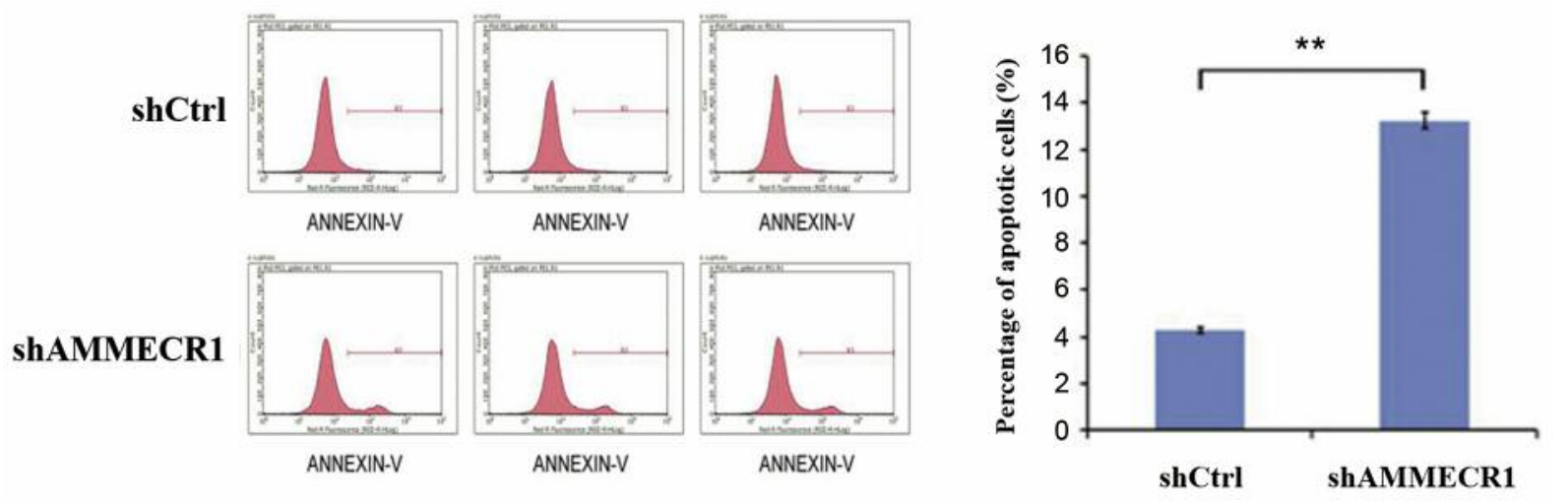

Figure 5. Apoptosis assessed with annexin V-allophycocyanin (APC) assay and flow cytometry. Apoptosis was significantly increased in cells transfected with Alport syndrome, mental retardation, midface hypoplasia, and elliptocytosis chromosomal region gene 1-specific short hairpin RNA ( shAMMECR1) compared to shCtrl-transfected cells. **Significantly different at $p<0.01$.

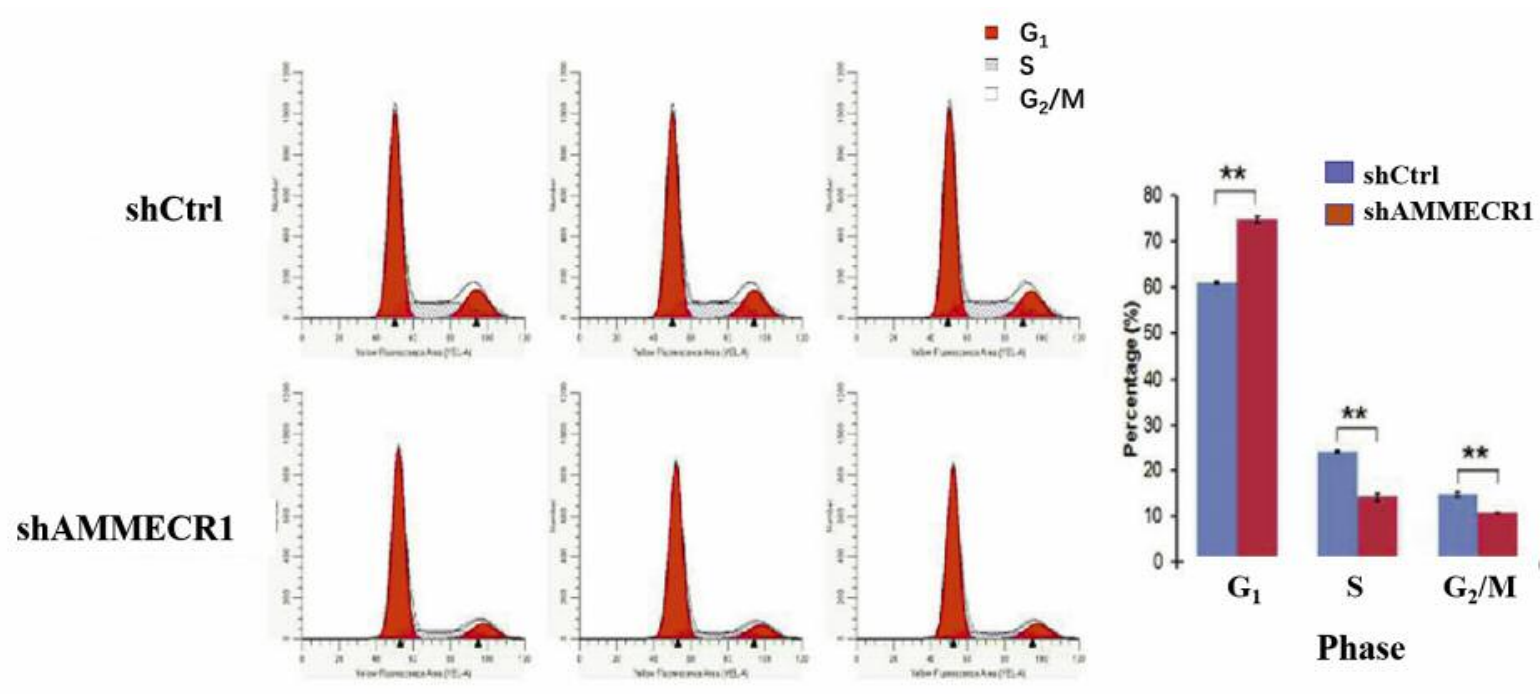

Figure 6. Cell-cycle distribution was examined with flow cytometry. Transfection of cells with short hairpin RNA against Alport syndrome, mental retardation, midface hypoplasia, and elliptocytosis chromosomal region gene 1 (shAMMECR1) led to a significant decrease in the percentage of cells in the $S$ and $G_{2} / M$ phases, and increase in the percentage of cells in the $G_{1}$ phase compared to shCtrl-transfected cells. **Significantly different at $p<0.01$.
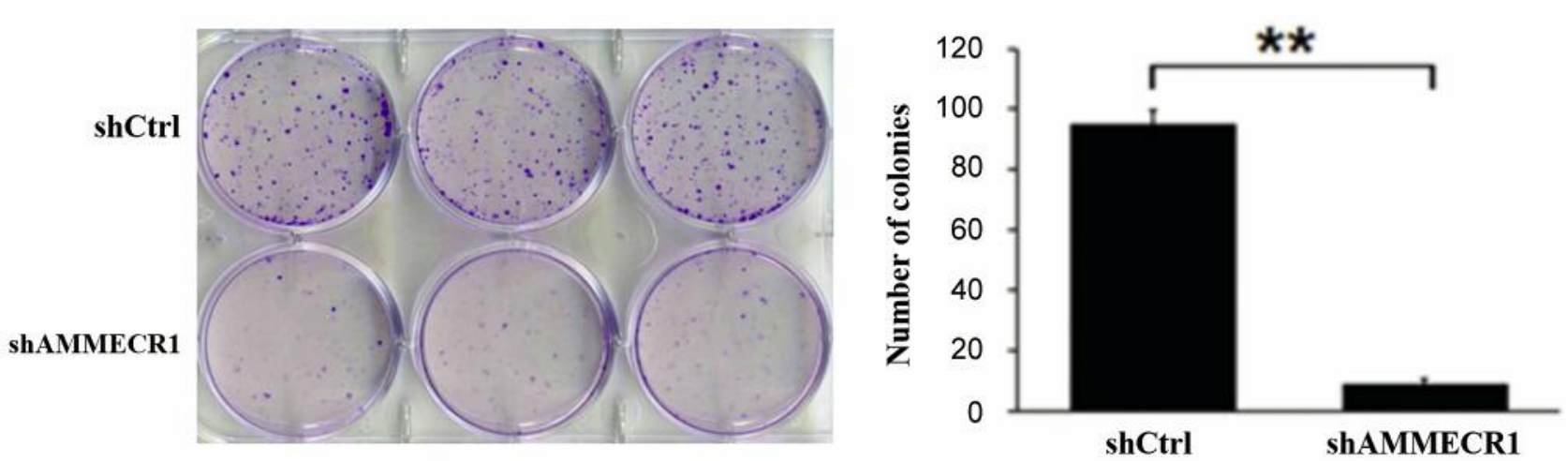

Figure 7. Colony formation assay with Giemsa staining. Alport syndrome, mental retardation, midface hypoplasia, and elliptocytosis chromosomal region gene 1-specific short hairpin RNA (shAMMECR1)-transfected cells had a significantly reduced number of cell colonies compared to shCtrl cells. **Significantly different at $p<0.01$. 


\section{Discussion}

The aim of this study was to investigate the role of AMMECRI in human lung cancer cell. AMMECRl expression was examined in several lung cancer cell lines. AMMECRI knockdown in lung cancer cells was performed to examine the role of $A M M E C R 1$ in lung cancer cell proliferation. This study showed, for the first time, that AMMECRI expression was increased in human lung cancer cell lines. Furthermore, $A M M E C R 1$ silencing was found to significantly inhibit lung cancer cell proliferation and colony formation, while significantly promoting apoptosis in vitro. A significant decrease in $\mathrm{G}_{2} / \mathrm{M}$-phase and S-phase, and increase in $\mathrm{G}_{1}$-phase cell populations were found in AMMECRI knockdown lungcancer cells. These results suggest that AMMECRI expression has a role in modulating proliferation of lung cancer cells.

There exist several limitations to the present study. Firstly, although four lung cell lines were examined for AMMECRI expression, only one was studied for its role in cancer cell proliferation and apoptosis. Therefore, more lung cancer cell lines need to be investigated in future studies. Secondly, only $A M M E C R 1$ silencing was performed in the present study. Future studies will examine the effects of overexpression of AMMECR1 on lung cancer cells. Thirdly, AMMCER1 expression will be investigated in lung cancer specimens from patients to further verify the association of AMMCERI expression with lung cancer.

In conclusion, this study demonstrated that AMMECR 1 may play a role in modulating proliferation in lung cancer cells.

\section{Conflicts of Interest}

None of the Authors have any conflict of interest in regard to this study.

\section{Authors' Contributions}

Haibo Ge contributed to the conception of the study; Ningchang Cheng and Xiaofeng Xu contributed significantly to analysis and article preparation; Jia Zhu performed the data analyses and wrote the article; Robert M. Hoffman and Zhijian Yang revised the article.

\section{Acknowledgements}

This study was supported by the National Natural Science Foundation of China (No. 81473609).

\section{References}

1 Shi Y and Sun Y: Medical management of lung cancer: Experience in China. Thorac Cancer 6: 10-16, 2015. PMID: 26273329. DOI: 10.1111/1759-7714.12168

2 Chen WQ, Zheng RS and Zeng HM: Epidemiology of lung cancer in China. Thorac Cancer 6: 209-215, 2015. PMID: 26273360. DOI: 10.1111/1759-7714.12169
3 Zheng M: Classification and pathology of lung cancer. Surg Oncol Clin N Am 25: 447-468, 2015. PMID: 27261908. DOI: 10.1016/j.soc.2016.02.003

4 Hirsch FR, Scagliotti GV, Mulshine JL, Kwon R, Curran WJ Jr., $\mathrm{Wu} \mathrm{YL}$ and Paz-Ares L: Lung cancer: Current therapies and new targeted treatments. Lancet 389: 299-311, 2016. PMID: 27574741. DOI: $10.1016 / \mathrm{S} 0140-6736(16) 30958-8$

5 Zhou HM: Research advances of AMMECR1. Biophysics 03: 16, 2015. DOI: 10.12677/biphy.2015.31001

6 Vitelli F, Piccini M, Caroil F, Franco B, Malandrini A, Pober B, Jonsson J, Sorrentino V and Renieri A: Identification and characterization of a highly conserved protein absent in the Alport syndrome (A), mental retardation (M), midface hypoplasia (M), and elliptocytosis (E) contiguous gene deletion syndrome (AMME). Genomics 55: 335-340, 1999. PMID: 10049589. DOI: 10.1006/geno.1998.5666

7 Meloni I, Vitelli F, Pucci L, Lowry RB, Tonlorenzi R, Rossi E, Ventura M, Rizzoni G, Kashtan CE, Pober B and Renieri A: Alport syndrome and mental retardation: clinical and genetic dissection of the contiguous gene deletion syndrome in Xq22.3 (ATS-MR). J Med Genet 39: 359-65365, 2002. PMID: 12011158. DOI: 10.1136/jmg.39.5.359

8 Oohashi F, Aga Y, Yukawa Y and Akama K: Novel in vivo system to monitor tRNA expression based on the recovery of GFP fluorescence and its application for the determination of plant tRNA expression. Gene 703: 145-152, 2019. PMID: 30940526. DOI: 10.1016/j.gene.2019.03.068

9 Pan Y, Shan W, Fang H, Guo M, Nie Z, Huang Y and Yao S: Sensitive and visible detection of apoptotic cells on annexin- $\mathrm{V}$ modified substrate using aminophenylboronic acid modified gold nanoparticles (APBA-GNPs) labeling. Biosens Bioelectron 52: 62-68, 2014. PMID: 24021657. DOI: 10.1016/j.bios.2013.07.057

10 Stinchcombe TE: Targeted therapies for lung cancer. Clin Adv in Hemat Oncol 170: 165, 2016. PMID: 27535394. DOI: 10.1007/978-3-319-40389-2_8

11 Sculier JP, Berghmans T and Meert AP: Advances in target therapy in lung cancer. Eur Respir Rev 24: 23-29, 2015. PMID: 25726551. DOI: 10.1183/09059180.00011014

12 Russo A, Rolfo C, Passiglia F and Rosell R: Targeted therapies for non-small cell lung cancer. In: Targeted Therapies for Solid Tumors. A Handbook for Moving Toward New Frontiers in Cancer Treatment. Russo A, Rosell R and Rolfo C (eds.). Humana Press. Springer. New York, pp. 89-101, 2015. DOI: 10.1007/978-1-4939-2047-1_9 